\title{
La predicción de la demanda en evaluación de proyectos*
}

\author{
Mar González Savignat \\ Universidad de Vigo
}

\author{
Anna Matas \\ Universitat Autònoma de Barcelona \\ Josep Lluís Raymond \\ Universitat Autònoma de Barcelona
}

\section{Resumen}

La predicción de la demanda juega un papel clave en la evaluación de proyectos. Sin embargo, la evidencia reciente indica que la predicción a largo plazo está sujeta a fuertes incertidumbres. Este artículo discute algunos aspectos relevantes relacionados con la predicción de la demanda a largo plazo y, en especial, la incertidumbre. En primer lugar, el artículo revisa los principales aspectos que la predicción de la demanda debe tomar en consideración para evitar errores que pueden distorsionar los resultados de la evaluación. A continuación, se analiza de forma sucinta las ventajas e inconvenientes de los modelos de demanda a efectos de predicción, con especial referencia a la predicción a largo plazo y a la elección modal. En tercer lugar, se caracterizan los errores de predicción y se presenta como ejemplo ilustrativo el impacto de la incertidumbre en la predicción a largo plazo para una autopista de peaje. Por último, se resumen los modelos de predicción de la demanda aplicados en España.

Palabras clave: modelos de demanda, predicción largo plazo, incertidumbre.

Clasificación JEL: C53, D61, R41.

\begin{abstract}
Demand forecasting is a key factor in project evaluation. However, according to recent empirical evidence, long-term predictions are subject to high levels of uncertainty. This paper discusses some relevant topics related to long run demand forecasting with special emphasis on uncertainty. Firstly, we offer a review of the main issues that demand forecasting should consider in order to avoid errors that might distort the result of the evaluation. Secondly, we analyze the pros and cons of alternative modelling approaches, particularly, long term forecast and modal split models. The paper goes on to describe the characteristics of the forecasting errors and provides, as an illustrative example, the impact of uncertainty on long term forecasting for a tolled motorway. Finally, we summarize the forecasting models used in Spain .
\end{abstract}

Keywords: demand models, long term forecasting, uncertainty.

JEL Classification: C53, D61, R41.

* Los autores agradecen la financiación recibida a través del proyecto PT2007-001-IAPP del CEDEX del Ministerio de Fomento y del proyecto ECO2008-05771 del Ministerio de Ciencia e Innovación. 


\section{Introducción}

La rentabilidad económica de un proyecto de transporte depende en gran medida de cuál sea la demanda futura de la infraestructura o modo de transporte evaluado. Los principales beneficios del proyecto se derivan de la reducción de los costes de tiempo y monetarios del viaje para los usuarios ya existentes y del aumento de la disposición a pagar correspondiente a los nuevos viajes inducidos por el proyecto. Por ello, es esencial realizar una predicción fiable de la demanda a lo largo de la vida útil del proyecto en la situación con y sin proyecto.

La predicción de la demanda requiere disponer de un modelo explicativo del comportamiento de los individuos en relación con los principales factores determinantes de la misma y, en particular, debe incorporar las posibles reacciones del usuario frente a un cambio en los costes. El enfoque metodológico adoptado dependerá de las características del proyecto o de la política a evaluar. En la evaluación de proyectos de inversión en infraestructura el modelo debe ser capaz de predecir los cambios en el flujo de viajes a largo plazo. Cuando un proyecto supone modificaciones significativas en la oferta de transporte es preciso predecir cómo responden los individuos en términos de incremento de la frecuencia de viaje, cambio de ruta, de modo de transporte y, en algunos casos, de destino. Otros proyectos tienen un impacto pequeño en los costes relativos de los modos de transporte de forma que sólo es necesario determinar los cambios de ruta y el aumento de frecuencia del tráfico existente. En la evaluación de políticas de transporte es más importante predecir la reacción de los individuos o empresas a cambios en las principales variables que determinan su elección. Por ejemplo, la introducción de un impuesto de congestión en áreas urbanas o un impuesto de circulación para los vehículos pesados según el coste marginal implica cambios en el comportamiento a corto plazo de los individuos o empresas. La evaluación de este tipo de políticas requiere un modelo que permita tener en cuenta que el comportamiento de los individuos no es homogéneo y que está influido por múltiples variables, cuyo efecto debe ser identificado en la ecuación de demanda. En este contexto los modelos estimados con datos microeconómicos muestran una mejor capacidad para simular políticas alternativas que los modelos agregados tradicionales.

No existe un único modelo de demanda que pueda ser recomendado para evaluar cualquier proyecto o política de transporte. Será necesario analizar en cada caso los impactos esperados del proyecto y buscar el modelo más adecuado entre los múltiples enfoques disponibles.

Los epígrafes siguientes analizan los principales elementos que debe contemplar un modelo de demanda, las técnicas econométricas más adecuadas, la incorporación de la incertidumbre en las predicciones a largo plazo y la situación en España. Debido a que no es posible cubrir en unas pocas páginas todo el rango de posibles enfoques metodológicos, el artículo está orientado a la predicción de la demanda en un contexto de evaluación de proyectos de inversión en infraestructura. En particular, se dirige a los proyectos interurbanos. Las actuaciones de transporte en medio urbano afectan, en general, a toda la red de transporte lo que exige modelos más sofisti- 
cados y adecuados al área de estudio. El elevado nivel de recursos que absorben las inversiones en proyectos interurbanos y su carácter irreversible justifican por si solos dedicar atención a su evaluación.

\section{Aspectos previos relacionados con la predicción de la demanda}

Un paso preliminar a la especificación del modelo es determinar correctamente el área geográfica afectada por el proyecto. Es bien conocido que el impacto de una infraestructura sobre el tráfico va más allá de la región directamente afectada y genera efectos desbordamiento sobre las áreas vecinas.

Un segundo aspecto que debe abordarse es si se estima un modelo multimodal o unimodal. El primero contempla de manera simultánea todos los modos de transporte, se trata de un modelo más completo, aunque mucho más complejo y exigente en cuanto a la información necesaria. Por ello, es menos frecuente y, en general, se utiliza en contextos con una fuerte interrelación entre medios de transporte. El modelo unimodal tiene como ventaja que permite una mayor riqueza en las variables incluidas en la ecuación de demanda y, por consiguiente, puede captar de manera más precisa los cambios en la red de transporte.

En tercer lugar es preciso determinar qué tipo de decisiones individuales se verán afectadas por el proyecto. Una inversión en transporte, en la medida que reduce el coste del trayecto, modifica cualquiera de las siguientes decisiones: generación de viajes, elección del destino, elección del modo de transporte, elección de ruta y día y hora del viaje. En la literatura de demanda de transporte estas decisiones se agrupan en las conocidas etapas de generación, distribución, elección modal y asignación a la red. Además, cuando se estime oportuno en la evaluación del proyecto, cada una de las anteriores decisiones o etapas puede segmentarse de acuerdo con el día u hora del viaje.

El modelo de generación estima el aumento del número de viajes como consecuencia de la reducción del coste generalizado ${ }^{1}$. Estos viajes adicionales proceden de nuevos usuarios o de un incremento en la frecuencia de los existentes. En décadas pasadas, la convención habitual era que el número de viajes se consideraba fijo y su crecimiento dependía sólo de las variables socioeconómicas. Sin embargo, a partir de la década de los años noventa la evidencia va en contra de la hipótesis de demanda totalmente inelástica respecto a los $\operatorname{costes}^{2}$. Con el fin de cuantificar el impacto de los incrementos en accesibilidad en el crecimiento del tráfico, el modelo de generación debe incluir, además de las variables socioeconómicas, las variables de coste generalizado. Esto sería lo apropiado, por ejemplo, para el caso de una inversión en una infraestructura nueva como el tren de alta velocidad. Por el contrario, el supuesto de matriz fija de viajes puede ser más fácilmente asumible en la

\footnotetext{
${ }^{1}$ En este artículo se entiende por coste generalizado los costes monetarios y los costes de tiempo asociados a cada viaje.

${ }^{2}$ Véanse GOODWIN (1996), NOLAND (2001), YAO y MORIKAWA (2006).
} 
evaluación de mejoras en algunas inversiones de carreteras. En aquellos casos que existan dificultades para desarrollar un modelo de generación robusto debido a la falta de información existente de costes monetarios y de tiempo, una aproximación alternativa es obtener el tráfico generado a partir de valores estándar de la elasticidad, siempre adoptando los resultados con prudencia dado que el comportamiento de la demanda depende de aspectos específicos del área geográfica analizada y de las características socioeconómicas de los individuos.

Además, a largo plazo, es posible que la mejora en la accesibilidad incentive cambios en la localización y descentralización de las actividades y residencias, dando lugar a un mayor número de viajes en la red y a una redistribución de los orígenes y destinos que deriva en un incremento en el número de kilómetros recorridos. La predicción del tráfico generado asociado a cambios en la localización requiere un modelo que relacione usos del suelo y transporte. Se trata de un modelo definido a escala regional que recoge la distribución espacial de actividades y los cambios en la accesibilidad del área inducidos por el proyecto. Sin embargo, dado que los individuos se localizan en función de sus preferencias por los distintos modos de transporte, la estimación econométrica de estos modelos se enfrenta a un problema de autoselección muestral difícil de resolver. Ello puede dar lugar una sobrepredicción de la demanda cuando se analiza los efectos de mejoras en la accesibilidad en la localización de las familias o empresas. Existe evidencia que cuando se controla por la autoselección, la relación entre usos del suelo y transporte es mucho menor (Small y Verhoef, 2007). Una modelización alternativa se basa en la construcción de modelos de simulación en áreas urbanas. Estos son modelos complejos que incluyen un amplio número de decisiones, aunque a costa de introducir supuestos muy simplificados sobre el comportamiento de los individuos para que sea computacionalmente tratable (ver, por ejemplo, Anas y Liu, 2007). En proyectos relacionados con cambios en la localización, la prudencia en la evaluación aconseja comparar los resultados de las predicciones con los efectos observados en proyectos similares.

La elección del destino del viaje depende de un amplio conjunto de variables, entre ellas, el coste de transporte. No obstante, la relevancia de esta última variable depende del motivo del viaje y de las características del individuo. Así, por ejemplo, en los viajes al trabajo o visitas a familiares, el destino es fijo, al menos en el corto y medio plazo. Por ello, es aconsejable estimar los modelos de distribución de viajes con datos individuales; sin embargo, no es habitual disponer de matrices origendestino suficientemente representativas con este tipo de datos. La dificultad de estimación y la consiguiente posibilidad de cometer errores explican que esta etapa a menudo se excluya del modelo de demanda.

Los modelos de elección modal gozan de una larga tradición en economía del transporte. Los costes relativos entre los modos de transporte son el principal determinante de las elecciones individuales de viaje. Cuando la inversión supone una reducción significativa en el coste generalizado de un servicio de transporte en relación con las existentes, es necesario cuantificar los cambios en el reparto modal. Un claro ejemplo de ello es el impacto de una nueva línea de alta velocidad sobre el 
transporte aéreo. En estos casos, un modelo de elección modal permite evaluar el desvío de viajes entre las distintas alternativas a partir de las características de los modos de transporte. Estas características son el precio, los costes de tiempo, la frecuencia y la fiabilidad del servicio. Así, por ejemplo, Asensio y Matas (2008) hallan que los individuos asignan una valoración a la variabilidad en el tiempo de viaje 2,5 veces superior al valor de un ahorro de tiempo en las autopistas de peaje en áreas metropolitanas. Por analogía cabe suponer que un resultado similar opere en el tren de alta velocidad frente al avión en España.

El modelo de demanda se cierra con la asignación de los viajes a la red. El modelo de asignación considera la decisión de elección de una ruta determinada en una red y calcula los costes de dicha ruta. Se trata, en general, de modelos matemáticos con un grado de sofisticación variable que va desde complejos modelos de simulación en áreas urbanas hasta la especificación de una sencilla relación velocidad-flujo en corredores interurbanos.

El modelo de asignación permite incorporar en la evaluación del proyecto los costes de congestión que aparecen a medida que el tráfico en una ruta aumenta. De hecho, un supuesto frecuente en la evaluación es que el coste generalizado se mantiene constante a lo largo de los años de vida del proyecto. Sin embargo, ignorar la capacidad máxima de una infraestructura puede generar errores muy importantes en la evaluación de los beneficios netos de un proyecto cuando no se prevén mejoras en la capa$\operatorname{cidad}^{3}$. Es preciso, pues, tener en cuenta que la relación entre demanda de transporte y calidad -medida en costes de tiempo- es bidireccional y requiere un procedimiento de cálculo iterativo entre el modelo de demanda y el modelo de asignación a la red.

Con el fin de que el modelo de demanda sea útil para la evaluación de proyectos de transporte, debe proporcionar resultados que puedan incorporarse en la evaluación. En este sentido, es necesario decidir el nivel de desagregación dependiendo del tipo de tráfico (por ejemplo, vehículos ligeros o pesados para carreteras, pasajeros de tráfico doméstico o internacional para aeropuertos, pasajeros y mercancías en ferrocarril) y el periodo de tiempo (día de la semana u hora del día). Cuanto más alto el detalle, más complejo será hallar el equilibrio entre la demanda y la red ofertada. Por ello, las decisiones de desagregación deberán tomarse con cautela y de acuerdo con las exigencias del problema y la calidad de los datos disponibles.

Además, los supuestos bajo los que se estima el modelo de demanda deben ser consistentes con los supuestos realizados en las medidas de bienestar. Por ejemplo, si la evaluación de un proyecto utiliza distintos valores del tiempo según el tipo de usuario (viaje de trabajo, viaje de ocio y otros) será necesario segmentar la demanda de acuerdo con el mismo criterio.

A efectos de evaluación el modelo de demanda debe permitir la distinción entre el tráfico existente antes de la inversión y el tráfico inducido por la misma. Este último comprende el tráfico desviado de otros modos de transporte y la generación de

\footnotetext{
${ }^{3}$ Véase MACKIE (1996).
} 
nuevos viajes como consecuencia de la reducción de costes derivada de la inversión. El tráfico desviado se obtiene directamente del modelo de elección modal. Respecto a la generación de nuevos viajes, en un contexto dinámico de crecimiento del tráfico es difícil distinguir entre los viajes derivados del crecimiento económico de aquellos que resultan de la reducción del coste generalizado. Una buena especificación del modelo de generación que contemple tanto los factores económicos y demográficos, como el coste generalizado del modo de transporte es imprescindible.

Una vez estimadas las distintas etapas del modelo de demanda, es necesario predecir su evolución para los años de vida del proyecto. El comportamiento de la demanda dependerá de la evolución de los factores económicos y demográficos asî como de la del coste generalizado. Dado que se desconoce el valor futuro de las variables explicativas, un paso previo es predecir su evolución para el mismo periodo temporal, lo cual introduce un fuerte elemento de incertidumbre en la predicción de la demanda. Por ello, es recomendable elegir con cautela y una cierta parquedad las variables explicativas en cada fase de la demanda.

\section{Los modelos de demanda}

La elección del modelo de demanda más adecuado a efectos de predicción deberá hacerse de acuerdo con las características del proyecto. En este apartado se analizan de forma sucinta tres de las principales decisiones que intervienen en la predicción de la demanda: la elección entre el enfoque agregado y el enfoque desagregado; los modelos de predicción a largo plazo y los modelos de elección modal ${ }^{4}$. Adicionalmente, se presenta la posibilidad de basar la predicción en valores estándares obtenidos a partir de experiencia previa.

\subsection{El enfoque agregado vs el enfoque desagregado}

Los modelos de predicción de demanda tienen su origen en la década de los años 50 en los Estados Unidos en el ámbito de evaluación de la capacidad de las infraestructuras viarias en las áreas metropolitanas. A grandes rasgos puede decirse que eran modelos ad-hoc estimados con datos agregados, es decir, datos que describen el comportamiento de grandes grupos de individuos. En las décadas posteriores se introducen mejoras progresivas en los modelos de demanda en cuatro ámbitos: su fundamentación en la teoría económica, las propias mejoras en las técnicas econométricas, el incremento en la capacidad de cálculo y la disponibilidad de bases de datos. Un punto de inflexión en la evolución de los modelos de demanda lo consti-

\footnotetext{
${ }^{4}$ Para una revisión reciente de la modelización de la demanda en trayectos interurbanos puede consultarse el estudio elaborado por SCOTT-WILSON, RAND EUROPE y H-G-A (2007) y la guía Transport Analysis Guide (TAG), del Departamento de Transporte del Reino Unido (2006).
} 
tuye el trabajo de McFadden, cuando a mediados de la década de los 70, desarrolla los fundamentos de los modelos de elección discreta en la teoría microeconómica del consumidor (McFadden, 1974, y Domencich y McFadden, 1975). A partir de este momento, la estimación de modelos de elección discreta en el ámbito del transporte se extiende de forma muy rápida con la ayuda de la progresiva implementación del software necesario en los programas econométricos estándares. Estos modelos estimados con datos individuales, de ahí la denominación de modelos desagregados en economía del transporte, se adaptan muy bien a la naturaleza claramente discreta de la demanda de transporte.

La generalización de los modelos «desagregados» se explica por sus ventajas frente los tradicionales modelos «agregados». En primer lugar, la base teórica de los modelos desagregados es más sólida en el sentido que no requiere suponer que todos los individuos tienen unas preferencias suficientemente similares para que su comportamiento quede reflejado por un individuo «promedio» de la población. En segundo lugar, permite una especificación de la función de demanda más rica dado que es posible medir mejor tanto las variables socioeconómicas de los individuos como los atributos de la oferta de transporte. Así, a cada individuo de la muestra se le asignan los valores de las variables a los que se enfrenta y no el promedio de la población. Ello permite medir el efecto de variables expresivas de la calidad como el tiempo, la frecuencia o la fiabilidad, variables que son difíciles, cuando no imposible, de incorporar en los modelos agregados. Además, es posible controlar por las características socioeconómicas del individuo y evitar que el efecto de la heterogeneidad de la muestra en las decisiones de transporte quede recogido por los atributos de la oferta. En tercer lugar, la introducción de un término aleatorio en la función de utilidad capta los gustos no observables de los individuos de tal manera que dos individuos con las mismas características socioeconómicas y que se enfrentan a los mismos atributos de la red de transporte pueden elegir alternativas distintas. En cuarto lugar, los modelos de elección discreta permiten estimar de forma interdependiente dos o más decisiones de transporte sin tener que suponer que éstas son secuenciales tal y como ocurre en los modelos con datos agregados. Por último, cabe destacar que las innovaciones en el campo de la microeconometría se han traducido en mejoras significativas de los modelos de demanda con datos individuales. Entre múltiples ejemplos de aplicaciones avanzadas de modelos de elección discreta destacamos el modelo de parámetros aleatorios o logit mixto que combina los coeficientes aleatorios con una estructura de error muy flexible para el componente aleatorio de la función de utilidad, de forma que tiene en cuenta la correlación entre alternativas (véase, por ejemplo Train, 2003). Este modelo se ha mostrado útil para evaluar el resultado de políticas de transporte que implican patrones complejos de sustitución entre alternativas o decisiones de viaje interrelacionadas. La contrapartida está, no obstante, en las dificultades de estimación, dado que su resolución sólo es factible mediante técnicas de simulación.

En resumen, las ventajas de los modelos desagregados ponen de manifiesto que este debe ser el enfoque a seguir cuando el objetivo de la predicción es evaluar cambios complejos en el comportamiento de los individuos como respuesta a políticas 
de transporte específicas. El éxito de las distintas familias de modelos de elección discreta reside en captar la sensibilidad de la demanda a los cambios propuestos. En determinados contextos, por ejemplo, en la evaluación de nuevos modos de transporte, son imprescindibles. Además, el desarrollo de técnicas econométricas avanzadas acompañado del correspondiente software ha permitido analizar cuestiones relativamente complejas del comportamiento individual. Es necesario, no obstante, recordar que la calidad de los datos limita el uso de estos modelos.

Sin embargo, en la predicción para un periodo temporal largo como exige la evaluación de inversiones en infraestructura, el enfoque con datos desagregados pierde parte de sus ventajas. Ello es debido a que para predecir es necesario agregar las probabilidades individuales obtenidas en el modelo. Cuando la muestra es representativa de la población, la demanda agregada puede obtenerse como la suma ponderada de las probabilidades individuales, donde las ponderaciones reflejan el peso de cada individuo en la población. No obstante, la predicción para un periodo temporal largo implica conocer la distribución de las distintas variables explicativas en la población para cada uno de los años de predicción. Esta tarea es difícilmente realizable en la evaluación de un proyecto de infraestructuras.

A su vez, los modelos estimados con datos agregados han mejorado su capacidad explicativa y de predicción. En primer lugar, las funciones de demanda con datos agregados también pueden estar fundamentadas en la teoría del consumidor y, cuando ello es así, ofrecen mejores resultados que las ecuaciones especificadas sin apoyo teórico. En segundo lugar, el desarrollo de la econometría ha beneficiado de forma significativa la estimación con datos agregados, en particular, el desarrollo del análisis de cointegración ha dotado de mayor rigurosidad las estimaciones con series temporales. Por último, cabe destacar que las funciones de demanda estimadas con series temporales permiten conocer el ajuste dinámico de los individuos a los cambios en la red, resultado difícil de conseguir con datos microeconómicos.

En conclusión, existe una amplia gama de modelos econométricos que permiten estimar la función de demanda a efectos de predicción. En las últimas décadas se han introducido mejoras de forma continua desde distintos ámbitos de tal manera que en la actualidad la rigurosidad en la estimación de la demanda puede quedar garantizada siempre que el modelo y la especificación del mismo se ajuste al objetivo del estudio y se reconozcan las limitaciones que a menudo imponen los datos.

\subsection{Predicción del tráfico a largo plazo}

Los cambios en la demanda a lo largo de la vida útil del proyecto dependen esencialmente del crecimiento esperado de las variables socioeconómicas y de los cambios en el coste generalizado del viaje. En ocasiones se utiliza un simple modelo tendencial que extrapola la tendencia pasada para predecir el tráfico futuro. Este modelo supone que el comportamiento pasado continuará en el futuro, hipótesis que claramente implica una simplificación excesiva y que descarta, entre otros, la generación de nuevos viajes y el cambio modal. 
Los modelos econométricos ofrecen una metodología adecuada para predecir el tráfico. El nivel de fiabilidad de las predicciones depende en gran medida de una buena especificación de la función de demanda, es decir, de la forma funcional y de las variables explicativas incluidas. En relación con la forma funcional es recomendable partir de una forma funcional flexible que incorpore las restricciones dictadas por la teoría económica e introducir las simplificaciones admisibles por los datos.

Las variables explicativas se dividen en dos grandes grupos. El primero está formado por variables socioeconómicas y de entorno, tales como la población, el PIB, el comercio exterior o el índice de producción industrial para el transporte de mercancías. El segundo grupo lo constituyen las características de la oferta de transporte, el precio y la calidad. La medición de la calidad es clave en la demanda de transporte, dado que la evidencia muestra que los individuos son más sensibles, en general, a la calidad que al precio. Algunas de las variables de calidad son medibles con relativa facilidad, por ejemplo, el tiempo y la frecuencia, mientras que otras, como el confort o la fiabilidad, resulta casi imposible incluirlas en una predicción a largo plazo.

Cuando el objetivo es predecir a largo plazo, los datos de series temporales constituyen una forma natural de estimar la función de demanda por su capacidad de captar el ajuste dinámico de los individuos a cambios en los atributos de la oferta. Es bien conocido que los individuos no se ajustan de forma inmediata a los cambios debido a la persistencia de hábitos, incertidumbre e información incompleta. Una especificación dinámica permite discernir el número de periodos necesarios para completar el cambio y la velocidad del ajuste. Así, es posible distinguir entre corto y largo plazo y evaluar el porcentaje del cambio que se completa en cada periodo.

El uso de series temporales nos obliga a ser cuidadosos con el proceso de estimación econométrica. En particular, si las series no son estacionarias, tal como ocurre con frecuencia, es preciso acudir a las técnicas de cointegración para evitar las regresiones espurias. El análisis de cointegración requiere un elevado número de observaciones. Es aconsejable muestras no inferiores a 30 años. El uso de datos trimestrales o mensuales, aunque sea una forma fácil de ampliar el número de observaciones, no aumenta el contenido de la información por el mismo factor dado que la variabilidad de la muestra es esencialmente la misma. Además, el uso de datos inferiores al periodo anual aumenta la dificultad de obtener las variables explicativas de la demanda.

En aquellas inversiones en infraestructura que sea necesario modelizar de manera específica los efectos de generación de tráfico los datos cross-section pueden ser una mejor alternativa. Una de las principales limitaciones de las series temporales es el reducido número de variables explicativas disponibles y su falta de variabilidad temporal. Por ejemplo, si una compañía ferroviaria incrementa sus tarifas de acuerdo con el IPC, la variación en términos constantes será nula, y por lo tanto será imposible determinar su efecto en la demanda. Las muestras cross-section aportan una mayor variabilidad en los datos con lo que mejoran los resultados de la estimación. 
Los datos cross-section pueden obtenerse a partir de matrices origen-destino para una única área geográfica o datos agregados para distintas unidades espaciales. En particular, los datos cross-section son adecuados para identificar el impacto de cambios en la accesibilidad a estaciones de transporte. Por ejemplo, son útiles para responder a preguntas del tipo ¿cuál será el tráfico atraído por una nueva estación de ferrocarril o un nuevo aeropuerto? Mientras que los factores de generación y atracción tienden a mantenerse constantes en el tiempo, los datos cross-section permiten captar la variabilidad a partir de una muestra de distintas unidades de observación en un momento del tiempo ${ }^{5}$.

El uso de datos de panel es una forma adecuada y elegante de aprovechar las ventajas de ambos tipos de datos. Un panel de datos está formado por un conjunto de unidades cross-section observadas a lo largo de un período temporal. Sus ventajas son varias y pueden resumirse en los tres siguientes elementos. En primer lugar, un aumento del tamaño de la muestra y de los grados de libertad de la estimación; en segundo lugar, permiten introducir un mayor número de variables explicativas y, por último, dado que, en general, la variación entre unidades cross-section es superior a la variación temporal, la varianza de los estimadores es menor y, en consecuencia, su fiabilidad es más alta.

Una especificación econométrica flexible podría permitir que todos los coeficientes estimados varíen en función de la unidad cross-section. Sin embargo, es habitual introducir restricciones de igualdad admitidas por los datos para incrementar la fiabilidad de las estimaciones. Su principal desventaja reside en la dificultad de construir la base de datos, aunque en el ámbito del transporte es posible disponer de datos de panel para la red de carreteras, pasajeros en aeropuertos y viajeros en los distintos servicios de ferrocarril.

\subsection{Predicción del tráfico desviado}

Las inversiones en infraestructuras de transporte que reducen de manera sustancial el coste de una alternativa de transporte generan un fuerte impacto en la distribución de los viajeros entre modos de transporte. Por ejemplo, de acuerdo con distintos estudios, alrededor de un 30 por 100 del tráfico de las líneas de tren de alta velocidad procede del avión. Por ello, los modelos de elección modal resultan imprescindibles para evaluar determinadas inversiones.

Un modelo de elección modal estima el cambio de comportamiento de los individuos cuando se modifica el coste generalizado de alguna de sus alternativas de transporte. De acuerdo con el razonamiento del apartado 3.1, los modelos de elección discreta constituyen el mejor enfoque posible. Estos modelos cuentan con una

\footnotetext{
${ }^{5}$ Un estudio de WARDMAN, LYTHGOE y WHELAN (2007) destaca las ventajas de las observaciones cross-section para interpretar el comportamiento de la demanda de ferrocarril.
} 
larga tradición en economía del transporte y muestran un elevado grado de fiabilidad en la estimación de la elasticidad de la demanda respecto los principales atributos de la oferta.

Las ventajas de los modelos de elección discreta se han detallado ya en un anterior apartado y cabe decir que las mejoras econométricas desarrolladas se han aplicado en gran medida a la elección modal. Así, siempre que se disponga de los datos adecuados, es posible modelizar procesos complejos de sustitución entre alternativas y solucionarlos mediante técnicas de simulación.

Para estimar un modelo de elección modal es necesario disponer de una base de datos que contemple las decisiones individuales entre alternativas e incluya las principales características socioeconómicas del individuo y los atributos de todas las alternativas disponibles para cada uno de ellos. Ciertamente, poder estimar con datos que recogen las «preferencias reveladas» de los individuos confiere un alto grado de fiabilidad a las estimaciones. No obstante, el uso de datos observados limita el número y tipo de decisiones que pueden modelizarse. En el ámbito del transporte el uso de datos procedentes de «preferencias declaradas» ha ampliado de forma significativa los ámbitos de estudio, especialmente en aquellos contextos en los que la alternativa de transporte que se evalúa o cuya demanda se intenta predecir, aún no está disponible para viajar. Con las preferencias declaradas se simula un contexto con distintos escenarios hipotéticos en los que los individuos declaran sus elecciones modales. Además de caracterizar las preferencias de los viajeros respecto a alternativas de viaje existentes o no, presenta importantes ventajas tales como que las variables explicativas no presentan errores de medición ni están correlacionadas y presentan además una mayor variabilidad que a menudo es difícil de obtener cuando se utiliza información de preferencias reveladas.

\subsection{Predicción de acuerdo con valores estándares}

Hay que tener presente que en algunas circunstancias será difícil disponer del contexto y los datos adecuados para llevar a cabo una estimación suficientemente consistente de una función de demanda. Algunos ejemplos de ello son la predicción de la demanda para un nuevo modo de transporte o los cambios en la localización de las actividades como resultado de una inversión. Este podría ser el caso de la construcción de una nueva red de vías metropolitanas, la construcción de un nuevo aeropuerto en una ciudad o el enlace de un puerto a la red de ferrocarril. Estas dificultades se traducen en errores en la predicción. En estos casos, una opción alternativa es confiar en los resultados observados en proyectos similares realizados en otros ámbitos geográficos. La recopilación de información acerca de los efectos que determinadas inversiones han tenido sobre el tráfico constituye una alternativa para aproximar el impacto sobre la generación de nuevos viajes y sobre el cambio modal. Por ejemplo, la rentabilidad de la inversión en líneas de alta velocidad es un tema sujeto a discusión. En relación con la demanda potencial para este modo, la eviden- 
cia disponible pone de manifiesto que de forma aproximada un 30 por 100 de la demanda procede del avión, un 20 por 100 del coche y un 30 por 100 es nuevo tráfico generado ${ }^{6}$. La evidencia presentada en el cuadro 1 muestra que las inversiones en carretera inducen alrededor de un 20 por 100 de nuevo tráfico. El Departamento de Transporte del Reino Unido aconseja usar un valor del 15 por $100^{7}$. Sin embargo, cabe tener cautela dado que los estudios con datos microeconómicos sugieren valores significativamente menores.

La incorporación de la información contenida en los estudios tomados como referencia puede realizarse a través de la elasticidad estimada. En economía del transporte existe una amplia evidencia empírica acerca de los valores de la elasticidad de la demanda respecto a las principales variables explicativas. Esta amplia evidencia otorga credibilidad a los valores hallados y, por consiguiente, a su aplicación para estimar resultados de cambios en la oferta de transporte. Dado que cada proyecto de transporte tiene unas características muy específicas, es necesario elegir elasticidades estimadas en estudios de similares características. El contexto concreto en el que haya que predecir la demanda es lo que determinará qué tipo de elasticidad es la más apropiada para cada caso.

No obstante, es necesario realizar una advertencia en relación con la utilización de la elasticidad para evaluar cambios en la demanda derivados de un proyecto de transporte. Dado que la elasticidad está calculada para variaciones marginales de una variable, es posible que este valor no se mantenga cuando se evalúan inversiones en transporte que generan cambios discretos importantes en el coste de transporte.

Como ilustración se presenta en los Cuadros 1 y 2 una recopilación de estudios que estiman las elasticidades de la demanda en carretera y en tren de alta velocidad, respectivamente.

\footnotetext{
${ }^{6}$ Véase KING, 1996; HENSHER, 1997; DE RUS y ROMAN, 2006, y VICKERMAN, 1996.

${ }^{7}$ DEPARTMENT OF TRANSPORT (2006).
} 
CUADRO 1

ELASTICIDADES Y TRÁFICO INDUCIDO EN LAS INVERSIONES EN CARRETERA

\begin{tabular}{|c|c|c|c|c|}
\hline Estudio & Contexto & $\begin{array}{l}\text { Elasticidad } \\
\text { kilómetros } \\
\text { de red }\end{array}$ & $\begin{array}{l}\text { Elasticidad } \\
\text { tiempo de viaje } \\
\text { o velocidad }\end{array}$ & $\begin{array}{c}\text { Impacto } \\
\text { adicional sobre } \\
\text { la demanda }\end{array}$ \\
\hline \multicolumn{4}{|c|}{ MODELOS DATOS AGREGADOS } & \\
\hline SACTRA (1994) & $\begin{array}{c}\text { Revisión } 151 \\
\text { proyectos } \\
\text { mejoras carreteras }\end{array}$ & - & - & $10,4 \%$ \\
\hline SACTRA (1994) & $\begin{array}{c}\text { Revisión } 85 \\
\text { proyectos } \\
\text { mejora congestión }\end{array}$ & - & - & $16,4 \%$ \\
\hline Goodwin (1996) & Carretera & - & $\begin{array}{l}\text { Tiempo de viaje } \\
-0.5 \text { corto plazo } \\
-0.9 \text { largo plazo }\end{array}$ & $\begin{array}{l}10 \% \text { corto plazo } \\
20 \% \text { largo plazo }\end{array}$ \\
\hline $\begin{array}{l}\text { Hansen y Huang } \\
\text { (1997) }\end{array}$ & $\begin{array}{l}\text { Carreteras estado } \\
\text { California }\end{array}$ & $\begin{array}{l}\text { 0,3-0,7 condados } \\
\text { 0,5-0,9 áreas } \\
\text { metropolitanas }\end{array}$ & $\begin{array}{l}- \\
-\end{array}$ & \\
\hline Heanue (1998) & & - & - & $6 \%-22 \%$ \\
\hline $\begin{array}{l}\text { Fulton et al., } \\
(2000)\end{array}$ & $\begin{array}{c}\text { Carreteras estados } \\
\text { California } \\
\text { Virginia, Maryland. } \\
\text { Datos nivel condados }\end{array}$ & $0,3-0,5$ corto plazo & - & - \\
\hline $\begin{array}{l}\text { Cervero y Hansen } \\
(2002)\end{array}$ & $\begin{array}{c}\text { Carreteras } \\
\text { California. Datos } \\
\text { ámbito condados }\end{array}$ & 0,56 & & \\
\hline Noland (2001) & $\begin{array}{c}\text { Carreteras estatales } \\
\text { EE.UU. }\end{array}$ & $\begin{array}{c}\text { Urbanas: } \\
0,44-0,51 \text { corto plazo } \\
0,81-1,0 \text { largo plazo }\end{array}$ & & $21,0 \%-26,5 \%$ \\
\hline Noland (2001) & $\begin{array}{c}\text { Carreteras estatales } \\
\text { EE.UU. }\end{array}$ & $\begin{array}{c}\text { Rurales: } \\
0,23-0,41 \text { corto plazo } \\
0,71-1,1 \text { largo plazo }\end{array}$ & & $21,0 \%-26,5 \%$ \\
\hline Cervero (2003) & $\begin{array}{l}\text { Carreteras, } \\
\text { California } \\
\text { corredores } \\
\text { suburbanos }\end{array}$ & - & $\begin{array}{c}\text { Velocidad } \\
0,24 \text { corto plazo } \\
0,64 \text { largo plazo }\end{array}$ & - \\
\hline \multicolumn{5}{|c|}{ MODELOS DATOS DESAGREGADOS } \\
\hline $\begin{array}{l}\text { Stratham et al. } \\
\text { (2000) }\end{array}$ & $\begin{array}{c}\text { Datos } \\
\text { individuales, } 48 \\
\text { áreas EE.UU. }\end{array}$ & 0,29 & & \\
\hline Barr (2000) & $\begin{array}{l}\text { Datos } \\
\text { individuales, } \\
\text { EE.UU. }\end{array}$ & & $-0,3-0,5-$ & $\begin{array}{c}\text { Positivo pero } \\
\text { inferior al hallado } \\
\text { en otros estudios }\end{array}$ \\
\hline
\end{tabular}

FUENTE: Elaboración propia. 


\section{CUADRO 2}

ELASTICIDAD DE LA DEMANDA PARA EL TREN DE ALTA VELOCIDAD

\begin{tabular}{|c|c|c|c|c|}
\hline Autor & Modo & Modelo & Elasticidad precio & Elasticidad tiempo \\
\hline Dodgson (1991) & TREN & $\begin{array}{l}\text { Revisión de } \\
\text { estudios previos }\end{array}$ & -1.0 & \\
\hline Nash (1991) & TREN & $\begin{array}{l}\text { Revisión de } \\
\text { estudios previos }\end{array}$ & $\begin{array}{l}-0.5 \text { (negocio) } \\
-1.25 \text { (ocio) }\end{array}$ & \\
\hline TRANSES (1991) & TREN & $\begin{array}{l}\text { Revisión de } \\
\text { estudios previos }\end{array}$ & & $\begin{array}{l}-1,6 \text { (negocio) } \\
-0,6 \text { (vacaciones) } \\
-1,1 \text { (otros) }\end{array}$ \\
\hline Wardman (1992) & TREN & $\begin{array}{l}\text { Revisión de } \\
\text { estudios previos }\end{array}$ & & $-1,3$ \\
\hline Nash (1997) & TREN & & & -1.3 \\
\hline Hensher (1997) & $\begin{array}{l}\text { AVIÓN } \\
\text { COCHE }\end{array}$ & $\begin{array}{l}\text { HEV Logit } \\
\text { (avión/TAV) } \\
\text { HEV } \\
\text { (coche/TAV) }\end{array}$ & $\begin{array}{l}-3.0 /-2.3 \text { (avión) } \\
-1,2 /-0,4 \text { (TAV) } \\
-0,16 \text { (coche) } \\
-0,47 /-0,8 \text { (TAV) }\end{array}$ & \\
\hline Nombela (2007) & $\begin{array}{l}\text { AVIÓN } \\
\text { TREN } \\
\text { BUS } \\
\text { COCHE }\end{array}$ & MNL (agregado) & $\begin{array}{l}-1,2 \\
-0,4 \\
-0,6 \\
-0,06\end{array}$ & $\begin{array}{l}-0,2 \\
-1,7 \\
-2,1 \\
-0,5\end{array}$ \\
\hline $\begin{array}{l}\text { González-Savignat } \\
\text { (2004) }\end{array}$ & $\begin{array}{l}\text { AVIÓN } \\
\text { COCHE }\end{array}$ & $\begin{array}{l}\text { Elasticidad modal } \\
\text { (avión/TAV) } \\
\text { Elasticidad modal } \\
\text { (coche/TAV) }\end{array}$ & $\begin{array}{l}-0,57 \text { (negocio) } \\
-1,34 \text { (ocio) } \\
-0,75 /-1,0 \text { (negocio) } \\
-1,14 /-1,6 \text { (ocio) }\end{array}$ & $\begin{array}{l}-1,20 \\
-0.92 /-1.20 \text { (negocio) } \\
-0,68-/-0,9\end{array}$ \\
\hline
\end{tabular}

FUENTE: Elaboración propia.

\section{Tratamiento de la incertidumbre en la predicción}

A pesar de las mejores incorporadas en la estimación de los modelos de demanda, las predicciones siguen sujetas a un importante nivel de incertidumbre que es preciso incorporar en la evaluación del proyecto para una correcta toma de decisiones. Algunos estudios recientes confirman la falta de acierto de las predicciones en transporte y aportan resultados de interés para la predicción que pueden resumirse en los siguientes puntos ${ }^{8}$. En primer lugar, la desviación estándar de las predicciones de demanda es elevada en la mayoría de estudios revisados lo que indica un alto nivel de incertidumbre. Por ejemplo, Flyvbjerg et al., (2006), a partir de una revisión internacional de más de 210 proyectos de inversión en carretera y

\footnotetext{
${ }^{8}$ Para una revisión más extensa, véase GONZÁLEZ-SAVIGNAT et al. (2009).
} 
ferrocarril, aportan evidencia de un elevado grado de error en las predicciones de la demanda más acusada en el caso de proyectos de ferrocarril. Para este último modo, los autores concluyen que en 9 de cada 10 proyectos la demanda está sobreestimada, con un valor promedio del 106\%; para los proyectos de inversión en carretera la predicción del tráfico es algo más fiable, aunque en un $50 \%$ de los casos la diferencia entre el valor predicho y el observado es superior a un $20 \%$. En segundo lugar, se observa un sesgo sistemático hacia la sobrepredicción en determinadas inversiones. Este es el caso de las inversiones en infraestructura de ferrocarril (Flyvbjerg et al., 2006, y Pickrell, 1989) o de las autopistas de peaje (Bain, 2009, y Vassallo y Baeza, 2007). Bain (2009) analiza los errores de predicción para más de 100 proyectos de inversión en autopistas de peaje y confirma un amplio grado de error en las predicciones de tráfico, junto con la existencia de un sesgo sistemático positivo. En promedio, las predicciones sobreestiman el tráfico del primer año entre un 20 y un 30\%. Vassallo y Baeza (2007), a partir de una muestra de 14 autopistas de peaje en España, hallan resultados muy similares a los de Bain, es decir, un sesgo sistemático en la predicción del tráfico que en promedio supera en un $35 \%$ el valor observado durante los tres primeros años de funcionamiento. En la mayor parte de los estudios revisados este sesgo se explica por un modelo de distribución modal excesivamente optimista acerca de la capacidad del ferrocarril o autopista de peaje para captar una parte de la demanda. En tercer lugar, los errores de predicción, medidos habitualmente en el primer año de funcionamiento de la infraestructura, se mantienen a lo largo del tiempo y, por consiguiente, no se deben a efectos imprevistos para el primer año de funcionamiento de la infraestructura. Por último, apuntar que los errores de predicción no guardan relación con el periodo de construcción y sólo se observa una relación muy débil con el coste del proyecto.

La magnitud observada en los errores de predicción obliga a incorporar la incertidumbre asociada a la predicción de la demanda en la evaluación de proyectos. La forma más usual de tratar la incertidumbre es construir distintos escenarios para las variables exógenas y predecir la demanda para cada uno de ellos. Sin embargo, este enfoque no contempla todas las fuentes de incertidumbre y, lo que es más importante, no ofrece la probabilidad de cada predicción alternativa.

Tal como es conocido, los errores en la predicción de la demanda provienen de dos fuentes. La primera está relacionada con la incertidumbre asociada a la predicción del valor futuro de las variables explicativas del tráfico a lo largo de la vida útil del proyecto (incertidumbre en los inputs). La segunda obedece a las perturbaciones o errores que afectan a la ecuación de demanda y que entre otros extremos reflejan las variables omitidas. De Jong et al., (2007), a partir de la revisión de un amplio número de estudios que identifican las distintas fuentes de incertidumbre en la predicción, concluyen que la incertidumbre en los inputs supera, generalmente, a la incertidumbre del modelo. Los estudios revisados por los citados autores hallan que los flujos de tráfico se sitúan entre el $18 \%$ y el $33 \%$ de la media para un intervalo de confianza del $95 \%$. 
Para fijar ideas, en un modelo de demanda lineal la varianza del error de predicción obedece a una expresión analítica sencilla. Así, si tenemos el siguiente proceso de generación de datos:

$$
y_{t}=x_{t}^{\prime} \cdot \beta+u_{t}
$$

la varianza del error de predicción « $\hat{\sigma}_{f} »$ puede expresarse como:

$$
\hat{\sigma}_{f}^{2}=x_{T+h}^{\prime} \cdot \operatorname{cov}(\hat{\beta}) \cdot x_{T+h}+\hat{\sigma}_{u}^{2}+\hat{\beta}^{\prime} \cdot \hat{\Sigma}_{v} \cdot \hat{\beta}
$$

Es decir, como la suma de los tres siguientes componentes:

- Varianza del error de predicción atribuible a la incertidumbre en el valor de los coeficientes del modelo: $x_{T+h}^{\prime} \cdot \operatorname{cov}(\hat{\beta}) \cdot x_{T+h}$.

- Varianza del error de predicción atribuible a la influencia de la perturbación aleatoria: $\hat{\sigma}_{u}^{2}$.

- Varianza del error de predicción atribuible al desconocimiento de los valores futuros de las variables explicativas: $\hat{\beta}^{\prime} \cdot \hat{\Sigma}_{v} \cdot \hat{\beta}$, siendo $《 \hat{\Sigma}_{v} »$ la matriz de varianzas y covarianzas de los errores predictivos en las variables explicativas.

En un modelo lineal tanto la matriz de varianzas y covarianzas de los coeficientes estimados beta como la varianza de la perturbación aleatoria pueden obtenerse a través de la correspondiente expresión analítica. La matriz de varianzas y covarianzas de los errores de predicción en las variables explicativas puede aproximarse por simulación o a través de procedimientos analíticos. Por ejemplo, si se empleasen modelos VAR para predecir los valores futuros de las variables explicativas, los propios modelos VAR estimados ofrecen esta información.

Una situación más frecuente es que el modelo no sea lineal. Una forma usual de proceder es emplear métodos de simulación a efectos de calcular la distribución de los errores predictivos. Consideremos el siguiente modelo de demanda:

$$
y=\Phi(X, \beta, \varepsilon)
$$

en el que la variable dependiente es una función de un conjunto de variables explicativas, un conjunto de coeficientes desconocidos $\beta$ y un término aleatorio $\varepsilon$. Los valores predichos se obtienen sustituyendo los términos desconocidos por sus respectivos valores estimados.

$$
\hat{y}=\Phi(\hat{X}, \hat{\beta}, \hat{\varepsilon})
$$

En el caso de realizar una simulación determinista, $\hat{\varepsilon}$ se fija en el valor esperado de $\varepsilon$, es decir en cero, $\hat{\beta}$ es el valor estimado de $\beta, \mathrm{y} \hat{X}$ es el valor asignado a las variables explicativas. 
En una simulación estocástica se supone que cada uno de los elementos de la ecuación [1] sigue una determinada distribución. Es decir:

$$
\begin{aligned}
X & \rightarrow \operatorname{Dist}\left(\hat{X}, \Sigma_{\hat{X}}\right) \\
\beta & \rightarrow \operatorname{Dist}\left(\hat{\beta}, \Sigma_{\hat{\beta}}\right) \\
\varepsilon & \rightarrow \operatorname{Dist}\left(0, \Sigma_{\varepsilon}\right)
\end{aligned}
$$

A partir de estas distribuciones es posible generar $\mathrm{M}$ realizaciones aleatorias distintas y resolver el modelo para cada una de ellas de manera que se obtienen $\mathrm{M}$ valores predichos para la variable dependiente. La distribución empírica de dichos valores permite calcular el valor esperado y la desviación estándar con lo cual es posible construir intervalos de confianza para las predicciones.

A modo de ejemplo se presenta los resultados de la predicción estocástica para una autopista de peaje en dos momentos del tiempo el año 2009 y el 2025. Las predicciones se han realizado a partir de un modelo de demanda no lineal estimado con datos de panel para el conjunto de autopistas de peaje en España. Para evaluar la incertidumbre total asociada a la predicción consideramos la distribución de $\hat{y}$, tráfico predicho, después de generar 1.000 realizaciones de $X, \beta, \varepsilon$. Para evaluar la incertidumbre asociada al modelo consideramos la distribución de $\hat{y}$ después de generar 1.000 realizaciones de $\beta, \varepsilon$; pero manteniendo fijos los valores de las variables explicativas en $\hat{X}$.

El Gráfico 1 muestra para el primer año predicho la distribución del error de predicción total y aquella debida sólo a la incertidumbre en el modelo. Para este primer año, las dos fuentes de error contribuyen aproximadamente por igual al error en las predicciones.

\section{GRÁFICO 1}

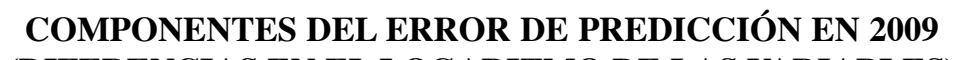
(DIFERENCIAS EN EL LOGARITMO DE LAS VARIABLES)

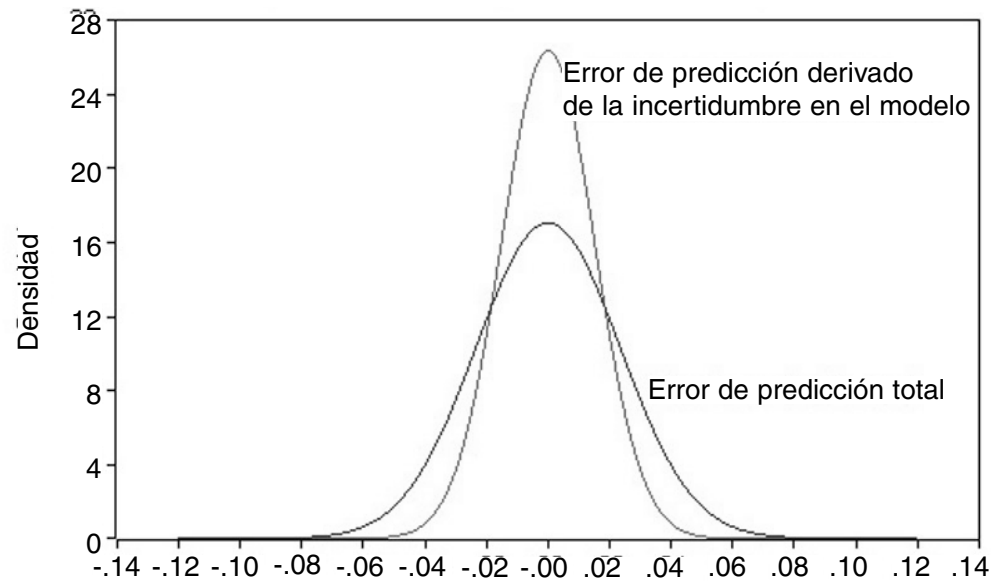

FUENTE: Elaboración propia. 
En el Gráfico 2 se observa el significativo aumento de la incertidumbre en la predicción a largo plazo. La desviación estándar del error de predicción pasa de 0,023 para el primer año predicho a 0,129 para el año 2025, siendo atribuible este aumento del error a la incertidumbre asociada al desconocimiento del valor futuro de las variables explicativas.

\section{GRÁFICO 2}
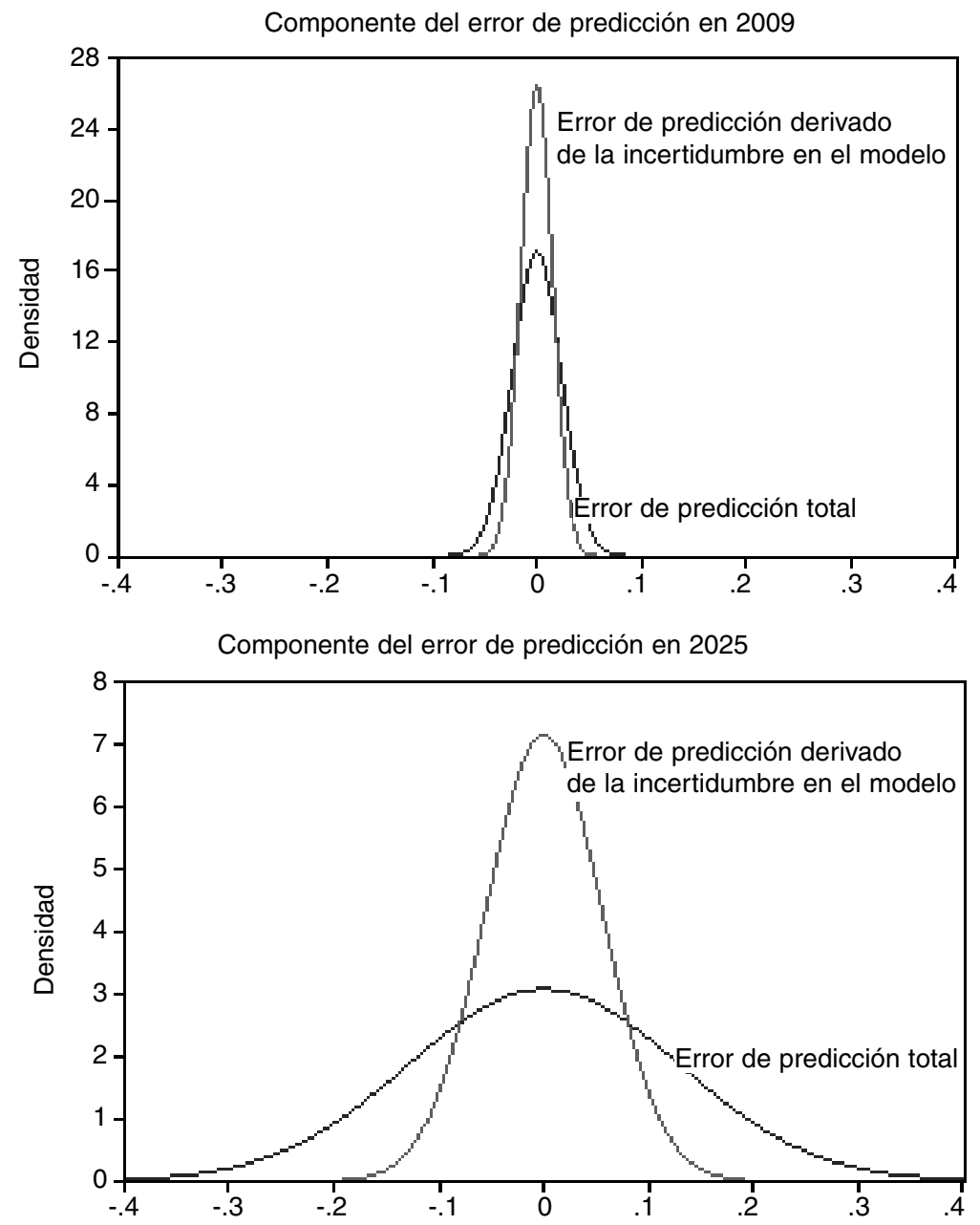

FUENTE: Elaboración propia.

En los modelos no lineales cabe destacar también que el predictor insesgado se obtiene promediando varias simulaciones estocásticas. De hecho, el resultado de la simulación determinista del modelo generalmente no coincide con el valor hacia el que tiende la media de las simulaciones estocásticas. 


\section{Predicción de la demanda de transporte en España}

En el marco de la política de transportes de la Unión Europea se plasma desde hace años una sensibilidad creciente acerca de la necesidad de incorporar la evaluación de inversiones de forma sistemática en el proceso de decisión de inversión pública. En este contexto, destaca el esfuerzo realizado a nivel institucional para la homogeneización de criterios en la evaluación tales como los documentos o manuales dedicados a la valoración monetaria de los costes y/o beneficios sociales. Sin embargo, a pesar de que son numerosos los manuales en este ámbito tanto de tipo general como relativos a aspectos más específicos o por modos de transporte, la predicción de la demanda se mantiene como una asignatura pendiente en la mayoría de las aportaciones. La razón principal es la dificultad para incorporarlo de forma práctica ya que, por todo lo expuesto anteriormente, resulta evidente que es una tarea complicada hacer extensiva una propuesta de predicción de demanda de transporte generalizable a contextos diferentes.

A pesar de esto, países como Reino Unido, Holanda, Dinamarca y Alemania han desarrollado modelos nacionales multimodales que permiten evaluar el efecto sobre la demanda de las políticas de transporte. Estos modelos ofrecen un marco de referencia excelente con el que comparar los resultados de modelos de demanda desarrollados para evaluar proyectos específicos. En España son relativamente numerosos los estudios sobre demanda de transporte, con un predominio de los modelos de elección discreta aplicados siempre a situaciones específicas y con dificultades para ser transferidos a otros ámbitos territoriales ${ }^{9}$.

Los organismos de la Administración Pública con competencias en el transporte han desarrollado distintos modelos para estimar y predecir la demanda. Se trata de modelos que contemplan un único modo de transporte y, en general, tienen por objetivo la predicción a medio y largo plazo de la demanda agregada a partir de variables socioeconómicas. Los modelos de demanda existentes para Puertos, Carreteras y Aeropuertos ofrecen una predicción del crecimiento de tráfico para un horizonte a medio y largo plazo que puede constituir un punto de partida para la predicción de la demanda del proyecto a evaluar y para conocer las principales variables explicativas de la demanda en cada modo de transporte. No obstante, no están diseñados para evaluar el impacto en la demanda de una determinada política o proyecto de transporte.

Por otro lado, en la medida que son modelos unimodales no permiten evaluar el impacto de políticas que afectan a más de una alternativa de transporte o que modifican de manera significativa el coste o calidad de una de ellas de tal manera que afecta la demanda de las alternativas sustitutivas. Es decir, no es posible cuantificar la demanda que cambia de modo de transporte. Por ejemplo, no sería adecuada esta aproximación para evaluar proyectos como la apertura de una línea de alta

\footnotetext{
${ }^{9}$ ROMÁN y ESPINO (2006) realizan un resumen de las principales aportaciones en España en el ámbito de la elección modal.
} 
velocidad o una nueva pista aeroportuaria. En segundo lugar, los modelos existentes no incorporan el coste generalizado del viaje lo cual impide contemplar la generación de nueva demanda como consecuencia de la reducción de dicho coste. Es cierto que para proyectos que suponen pequeños cambios del coste generalizado, es posible aplicar valores medios de las correspondientes elasticidades y predecir la demanda resultante según el comportamiento futuro de las variables socioeconómicas. Sin embargo, esta forma de proceder no es la adecuada cuando deben evaluarse proyectos como la apertura de una línea de alta velocidad o una nueva pista aeroportuaria.

Un aspecto imprescindible para realizar un estudio particular de una inversión en transporte es obtener datos desagregados o detallados, recurriendo para ello al organismo competente o a los propios operadores. Aquí aparece la primera dificultad; las bases de datos existentes en España sobre transporte y movilidad no están sistematizadas, ni centralizadas en ningún organismo de transporte o asociado que faciliten su accesibilidad. Además, el tipo de información que puede obtenerse a partir de ellas es muy limitada si se pretende utilizar en la predicción de la demanda. Una de las mayores carencias es la ausencia de matrices origen-destino de los viajes, tanto con datos de flujos agregados de tráfico como con datos individuales, para cualquier modo de transporte. Respecto a los datos de flujos de viajes, es recomendable presentarlos con una mayor desagregación en origen-destino o tipo de usuario. Este nivel de desagregación es fundamental para estimar modelos de demanda que sean útiles en la predicción y, por lo tanto, en la evaluación de inversiones.

Por lo tanto, se destaca la necesidad de mejorar y completar las bases de datos para poder estimar y valorar de forma más precisa los cambios en la demanda de transporte, aspecto en el que España presenta importantes carencias al comparar con otros países y que, sin embargo, constituye un elemento fundamental en evaluación de proyectos de transporte ya que es el que sustenta la rentabilidad de una inversión.

\section{Consideraciones finales}

La predicción de la demanda es un elemento imprescindible para evaluar la rentabilidad de un proyecto de transporte. Los avances en la metodología para estimar la demanda de transporte permiten seleccionar entre distintos enfoques alternativos aquel que mejor se ajusta a las características del proyecto a evaluar. No existe ni un modelo ni un enfoque metodológico que predomine sobre los demás desde todos los puntos de vista. No obstante, sí que existe un modelo que será el más adecuado para cada proyecto específico. La utilización de datos individuales es aconsejable siempre que sean previsibles cambios significativos en la selección de las alternativas de transporte por parte del usuario. Para la predicción del tráfico a largo plazo los modelos con datos agregados constituyen seguramente la mejor opción. En ambos enfoques metodológicos es recomendable fundamentar las ecuaciones de demanda 
en la teoría económica y controlar por los diferentes problemas econométricos que surgen en la estimación para minimizar los errores de predicción. Una atención especial merece la evaluación de nuevas infraestructuras por el riesgo de sobrepredicción de la demanda. El análisis de la evidencia disponible muestra que existe un sesgo positivo y sistemático en la predicción de la demanda para inversiones en ferrocarril y autopistas de peaje. Una pregunta relevante es por qué se da tal sesgo. Podría ser que se tratase de predicciones voluntaristas. En estos casos la selección y especificación de un modelo de demanda debe ser especialmente rigurosa y los resultados deben compararse con la experiencia en proyectos similares.

La presencia de incertidumbre en las predicciones es en cualquier caso inevitable y debe ser incorporada en la evaluación del proyecto. Una forma adecuada de tener en cuenta la incertidumbre es la construcción de intervalos de confianza para las estimaciones. Esta opción es relativamente fácil de implementar a partir de la estimación de un modelo econométrico.

Por último, apuntar que la situación en España dista de aquella disponible en otros países europeos. La recomendación sería avanzar hacia la construcción de modelos de demanda más completos que contemplen la generación de nuevos viajes, el cambio en la matriz de origen - destino y el cambio en el reparto modal consecuencia de una variación en el coste generalizado del viaje. Para ello es imprescindible la creación de una amplia base de datos homogénea para los distintos modos de transporte.

\section{Referencias bibliográficas}

[1] ANAS, A.y LIU, Y. (2007): «A regional economy, land use, and transportation model: Formulation, design, and testing», Journal of Regional Science, 47, 415-455.

[2] ASENSIO, J. y MATAS, A. (2008): «Commuters' valuation of travel time variability», Transportation Research, E, 44, 1074-1085.

[3] BAIN, R. (2009): «Error and optimism bias in toll road traffic forecasts», Transportation, 36, 469-482.

[4] BARR, L.C. (2000): «Testing for the significance of induced highway travel demand in metropolitan areas», Transportation Research Record, 1706, 1-8.

[5] CERVERO, R., y HANSEN, M. (2002): «Induced travel demand and induced road investment: a simoultaneous-equation analysis», Journal of Transport Economics and Policy, 36, 469-490.

[6] CERVERO, R., (2003): «Road expansion, urban growth, and induced travel», APA Journal, 69, 145-163.

[7] DE JONG, G.; DALY, A.; PIETERS, M.; MILLER, S.; PLASMEIJER, R., y HOFMAN, F. (2007): «Uncertainty in traffic forecasts: literature review and new results for The Netherlands», Transportation, 34, 375-395.

[8] DE RUS, G., y ROMÁN, C. (2006): «Análisis económico de la línea de alta velocidad Madrid-Barcelona», Revista de Economía Aplicada, 42. 
[9] DEPARTMENT OF TRANSPORT (2006). Transport Analysis Guide (TAG), Reino Unido.

[10] DOMENCICH, T.A., y McFADDEN, D. (1975): Urban Travel Demand: A Behavioral Analysis, North Holland.

[11] FLYVBJERG, B.; HOLM, M.; SKAMRIS, K., y BUHL, S. L. (2006): «Inaccuracy in Traffic Forecasts», Transport Reviews, 26:1, 1-24.

[12] FULTON, L.M. et al. (2000): «A statistical analysis of induced travel effects in the US mid-Atlantic region», Journal of Transportation and Statistics, 3, 1-14.

[13] GONZÁLEZ-SAVIGNAT, M.; MATAS, A.; RAYMOND, J.L., y RUIZ, A. (2009): «Predicting the demand: Uncertainty analysis and prediction models in Spain». Working Paper, Economic Evaluation of Transportation Projects.

[14] GOODWIN, P.B., (1996): «Empirical evidence on induced traffic, a review and synthesis», Transportation, 23, 35-54.

[15] HANSEN, M., y HUANG, Y. (1997): «Road supply and traffic in California urban areas», Transportation Research, A, 31, 205-218.

[16] HEANUE, K. (1998): «Higway capacity and induced travel: issues, evidence and implications». Transportation Research Circular, 481, TRB.

[17] HENSHER, D.A. (1997): «A Practical Approach to Identifying the Market Potential for High-Speed Rail: A Case Study in the Sydney-Canberra Corridor», Transportation Research, A, 31, 431-446.

[18] KING, J. (1996): «Economic significance of high speed rail», ITS Working Paper Series, The Institute of Transport Studies, The University of Sydney.

[19] MACKIE, P. (1996): «Induced traffic and economic appraisal», Transportation, 23, 103-119.

[20] McFADDEN, D. (1974): «The measurement of Urban Travel Demand», Journal of Public Economics, 3, 303-328.

[21] NOLAND, R.B. (2001): «Relationships between highway capacity and induced vehicle travel», Transportation Research, A, 35, 47-72.

[22] PICKRELL, D.H. (1989): Urban rail transit projects: forecasts vs actual ridership and costs. Department of Transportation, Transportation System Center, Cambridge, MA.

[23] ROMAN, C., y ESPINO, R. (2006): «La modelización de la demanda de transporte», Economistas, 110, 89-97.

[24] SACTRA (1994): Trunk roads and the generation of traffic. Dpt. of Transport, Standing Advisory Committee on Trunk Road Assessment, Londres.

[25] SCOTT-WILSON, RAND EUROPE y H-G-A, (2007): Modelling Langer distant demand for travel. Feasibility study. Final Report, Department of Transport.

[26] SMALL, K., y VERHOEF, E.K. (2007): Urban Transport Economics, Routledge. Capítulo 2.

[27] STRATHMAN, J.G. et al. (2000): Analysis of induced travel in the 1995 NPTS, Working Paper, Center for Urban Studies, Portland State University.

[28] TRAIN, K.E. (2003): Discrete choice methods with simulation, Cambridge University Press, Reino Unido.

[29] VASSALLO, J. y BAEZA, M. (2007): «Why Traffic Forecasts in PPP Contracts Are Often Overestimated?» Research Paper, Final Draft, EIB University Research Sponsorship Programme, European Investment Bank, Luxembourg. 
[30] VICKERMAN, R. (1997): «High-speed rail in Europe: experience and issues for future development», The Annals of Regional Science, 31, 21-38.

[31] WARDMAN, M.; LYTHGOE, W., y Whelan, G. (2007): «Rail passenger demand forecasting: cross-sectional models revisited», en Dennis y Talley (eds.), Railroad Economics, Research in Transportation Economics, vol. 20, Elsevier Ltd., 119-152.

[32] YAO, E., y MORIKAWA, T. (2005): «A study of an integrated intercity travel demand model», Transportation Research Part A, 39. 